\title{
Effectiveness of Whole, Inactivated, Low Pathogenicity Influenza A(H7N9) Vaccine against Antigenically Distinct, Highly Pathogenic H7N9 Virus
}

\section{Masato Hatta, Gongxun Zhong, Shiho Chiba, Tiago J.S. Lopes, Gabriele Neumann, Yoshihiro Kawaoka}

The recent emergence of highly pathogenic influenza A(H7N9) variants poses a great risk to humans. We show that ferrets vaccinated with low pathogenicity $\mathrm{H} 7 \mathrm{~N} 9$ virus vaccine do not develop severe symptoms after infection with an antigenically distinct, highly pathogenic H7N9 virus. These results demonstrate the protective benefits of this $\mathrm{H} 7 \mathrm{~N} 9$ vaccine.

$\mathrm{L}$ ow pathogenicity influenza $\mathrm{A}(\mathrm{H} 7 \mathrm{~N} 9)$ viruses, which cause mild or asymptomatic disease in poultry, have caused $\geq 1,564$ human infections since March 2013, with a case-fatality rate of $\approx 40 \%(1-5)$. Recently, highly pathogenic $\mathrm{H} 7 \mathrm{~N} 9$ viruses, characterized by multiple basic amino acids at the cleavage site of their hemagglutinin (HA) protein, have emerged. More than 750 cases of human H7N9 infections in 2017 (6) and the emergence of highly pathogenic H7N9 viruses emphasize the need for effective vaccines against low pathogenicity and highly pathogenic H7N9 viruses. We examined whether a World Health Organization (WHO) candidate vaccine based on a low pathogenicity H7N9 influenza virus would protect ferrets against an antigenically distinct, highly pathogenic H7N9 influenza virus.

\section{The Study}

We generated a recombinant virus (HK125-HYPR8) that possesses the HA and neuraminidase (NA) genes of a low pathogenicity WHO-recommended H7N9 candidate vaccine virus (A/Hong Kong/125/2017 [7]) and the remaining genes from a high-yield A/Puerto Rico/8/34 (PR8) vaccine backbone virus (8). The HK125-HYPR8 virus was inactivated with $\beta$-propiolactone and purified through sucrose gradient ultracentrifugation.

We vaccinated 5-month-old female ferrets (6 per group) that were serologically negative for currently circulating

Author affiliations: University of Wisconsin-Madison, Madison, Wisconsin, USA (M. Hatta, G. Zhong, S. Chiba, T.J.S. Lopes, G. Neumann, Y. Kawaoka); University of Tokyo, Tokyo, Japan (Y. Kawaoka)

DOI: https://doi.org/10.3201/eid2410.180403 human influenza viruses with $15 \mu \mathrm{g}$ of HA of inactivated whole HK125-HYPR8 virions without adjuvant (Group 1) or mixed at a 1:1 ratio with AddaVax adjuvant (InvivoGen, San Diego, CA, USA), a squalene-based oil-in-water nanoemulsion similar to MF59 (9) (group 2); control animals received phosphate-buffered saline (group 3) or adjuvant (group 4) (Figure 1, panel A). All animals were vaccinated intramuscularly in both hind legs twice, 28 days apart.

Twenty-eight days after the second immunization, we intranasally challenged ferrets with $10^{6}$ PFUs of highly pathogenic H7N9 rGD/3-NA294R virus (a neuraminidase inhibitor-sensitive subpopulation of highly pathogenic A/ Guangdong/17SF003/2016 H7N9 virus) (10). These vaccine and challenge viruses belong to the Yangtze River Delta lineage of H7N9 viruses, which is responsible for recent infections of humans with highly pathogenic H7N9 viruses (6). However, A/Hong Kong/125/2017 and the A/Guangdong/17SF003/2016 challenge virus differ antigenically (11) (online Technical Appendix Table 1, https:// wwwnc.cdc.gov/EID/article/24/10/18-0403-Techapp1.pdf).

We monitored clinical signs, body weight, and body temperature daily for 14 days and collected throat and nasal swab specimens every day until day 7 postchallenge. On day 4 postchallenge, we euthanized 3 ferrets from each group and collected organs (lung, trachea, nasal turbinates, olfactory bulbs, and brain tissues pooled from anterior and posterior brain sections) for virus titration. We conducted statistical analysis of hemagglutinin inhibition (HI) titers, virus titers in swab and organ samples, and bodyweight and temperature changes among groups (online Technical Appendix Tables $2-21)$. We defined statistical significance as $p<0.05$.

After 1 immunization, HI titers were significantly lower in the ferrets immunized with nonadjuvanted HK125HYPR8 vaccine than in those immunized with AddaVaxadjuvanted HK125-HYPR8 vaccine $(\mathrm{p}=0.038$; Figure 1 , panel B; online Technical Appendix Table 2); however, after 2 immunizations, ferrets vaccinated with or without adjuvant (groups 1 and 2) developed high HI titers against HK125HYPR8 virus. Vaccination with HK125-PR8 vaccine did not elicit measurable HI titers against the rGD/3-NA294R challenge virus after the first immunization but elicited reasonably high titers after the second immunization (Figure 1, panel B). After challenge with highly pathogenic $\mathrm{H} 7 \mathrm{~N} 9$ virus, 
nonvaccinated ferrets (groups 3 and 4) became lethargic, experienced diarrhea, and lost appetite and bodyweight on days 2-6 postinfection (online Technical Appendix Figure), whereas vaccinated ferrets showed no noticeable symptoms. In addition, nonvaccinated ferrets demonstrated statistically higher body temperature than vaccinated ferrets on days 1,2 , 3,5 , and 6 postchallenge (online Technical Appendix Figure, Table 5). One ferret in group 3 and 2 ferrets in group 4 had to be euthanized on days 6-8 postinfection (Figure 1, panel C) because of severe symptoms (neurologic signs or inability to remain upright). In contrast, none of the vaccinated ferrets had any symptoms, indicating a protective effect of the low pathogenicity H7N9 vaccine against the challenge virus.

Analysis of throat and nasal swab samples demonstrated replication of highly pathogenic challenge virus in all ferrets (Figure 2, panel A). However, virus titers started to decline in vaccinated ferrets by day 3 postchallenge, and the infection was resolved by day 5 postchallenge; in contrast, nonvaccinated ferrets continued to shed high titers of challenge virus 4-7 days postchallenge. The virus titers in nasal swab samples on days $1,3,4,5,6$, and 7 postchallenge and those in throat swab samples on days 1-7 postchallenge from nonvaccinated ferrets were significantly higher than those in vaccinated ferrets (online Technical Appendix Table 10). Thus, vaccination with HK125-HYPR8 virus led to reduced replication of the challenge virus in the upper respiratory tract of infected ferrets.

On day 4 postinfection, we euthanized 3 animals per group and determined virus titers in organs. We also assessed virus titers in organs of ferrets that were euthanized because of severe disease symptoms. In nonvaccinated ferrets, we detected high titers of virus in respiratory organs; in addition, we recovered virus from the olfactory bulbs or pooled samples from anterior and posterior sections of
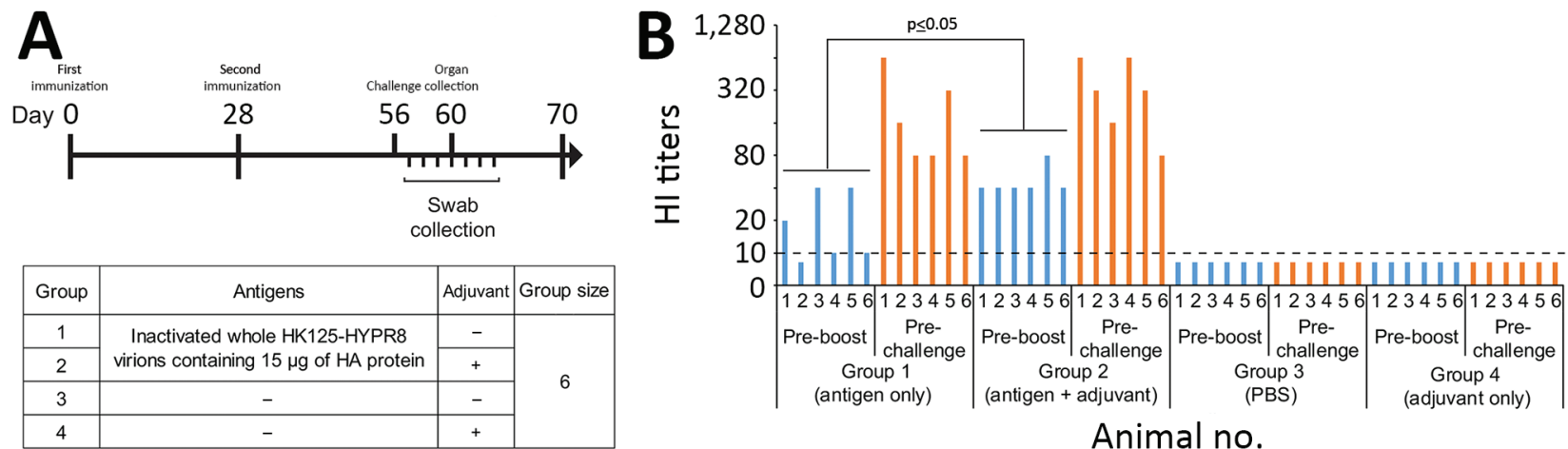

Animal no.
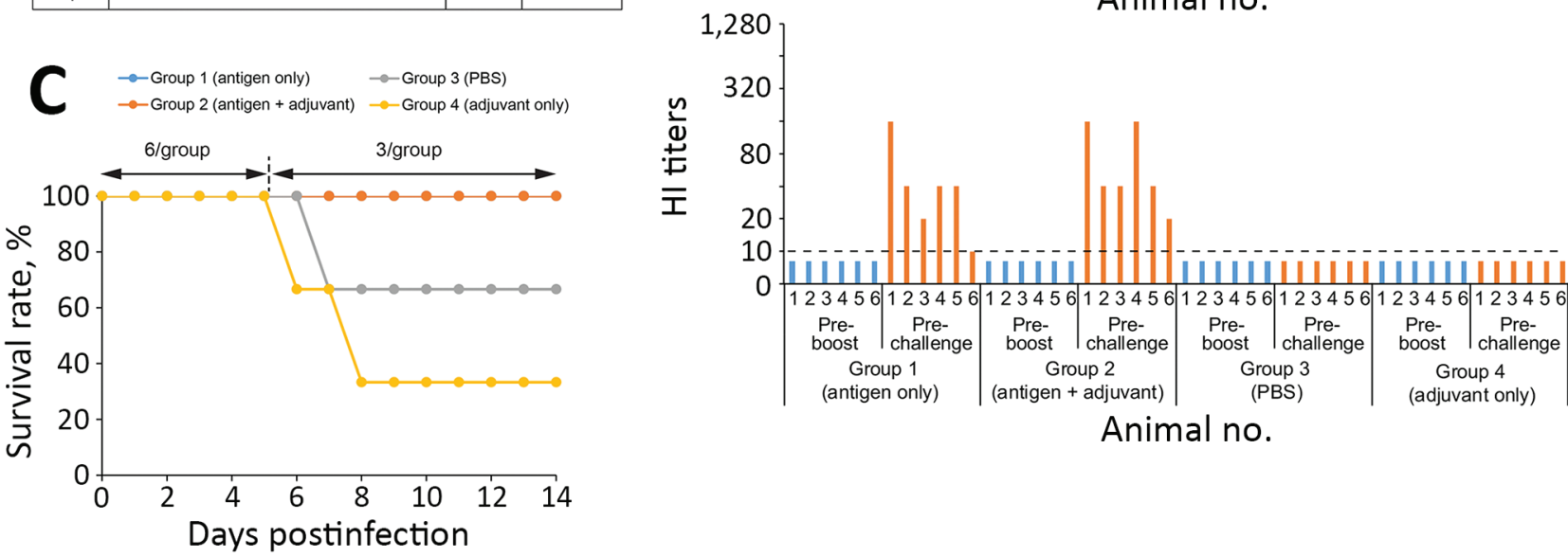

Figure 1. Study design, $\mathrm{HI}$ titers after vaccination, and survival rates of vaccinated and nonvaccinated ferrets challenged with highly pathogenic influenza A(H7N9) virus. A) Study design. Six ferrets per group were immunized with inactivated whole HK125-HYPR8 virions containing $15 \mu \mathrm{g}$ of HA protein without (group 1) or with adjuvant (group 2); control animals were vaccinated with PBS (group 3) or adjuvant (group 4). Animals were vaccinated intramuscularly twice 28 days apart. Twenty-eight days after the second immunization, ferrets were challenged with highly pathogenic H7N9 rGD/3-NA294R virus. Throat and nasal swab specimens were collected on days 1-7 postchallenge; 3 animals per group were euthanized on day 4 postchallenge to assess virus titers in organs. B) $\mathrm{HI}$ titers after vaccination. $\mathrm{HI}$ assays were performed against HK125-HYPR8 (upper panel) and rGD/3-NA294R (lower panel) with ferret sera collected before the second immunization (preboost) and before challenge (prechallenge). Statistical significance was determined as described in the online Technical Appendix (https://wwwnc.cdc.gov/EID/article/24/10/18-0403-Techapp1.pdf). C) Survival rates. Survival was monitored for 14 days after challenge. Because 3 ferrets were euthanized on day 4 postchallenge for organ sampling, the survival rate was calculated on the basis of a group size of $n=3$ thereafter. HA, hemagglutinin; HI, hemagglutination inhibition; PBS, phosphate-buffered saline. 
the brains of 7 of the 9 animals tested (Figure 2, panel B). In vaccinated ferrets, we detected virus in the nasal turbinates of 4 of 6 animals and in the olfactory bulbs of 2 of 6 animals. We recovered no virus from the tracheas, lungs, or pooled samples from anterior and posterior brain sections (Figure 2, panel B), indicating that vaccination with HK125-HYPR8 prevented challenge virus replication in the lower respiratory organs.

\section{Conclusions}

We report the effectiveness of a whole, inactivated, low pathogenicity H7N9 vaccine against an antigenically distinct, highly pathogenic H7N9 virus in a ferret model. Vaccination prevented challenge virus replication in the lower respiratory organs, led to faster virus clearance in the upper respiratory organs, and prevented severe disease and death in ferrets, although the HI titers against the rGD/3-NA294R
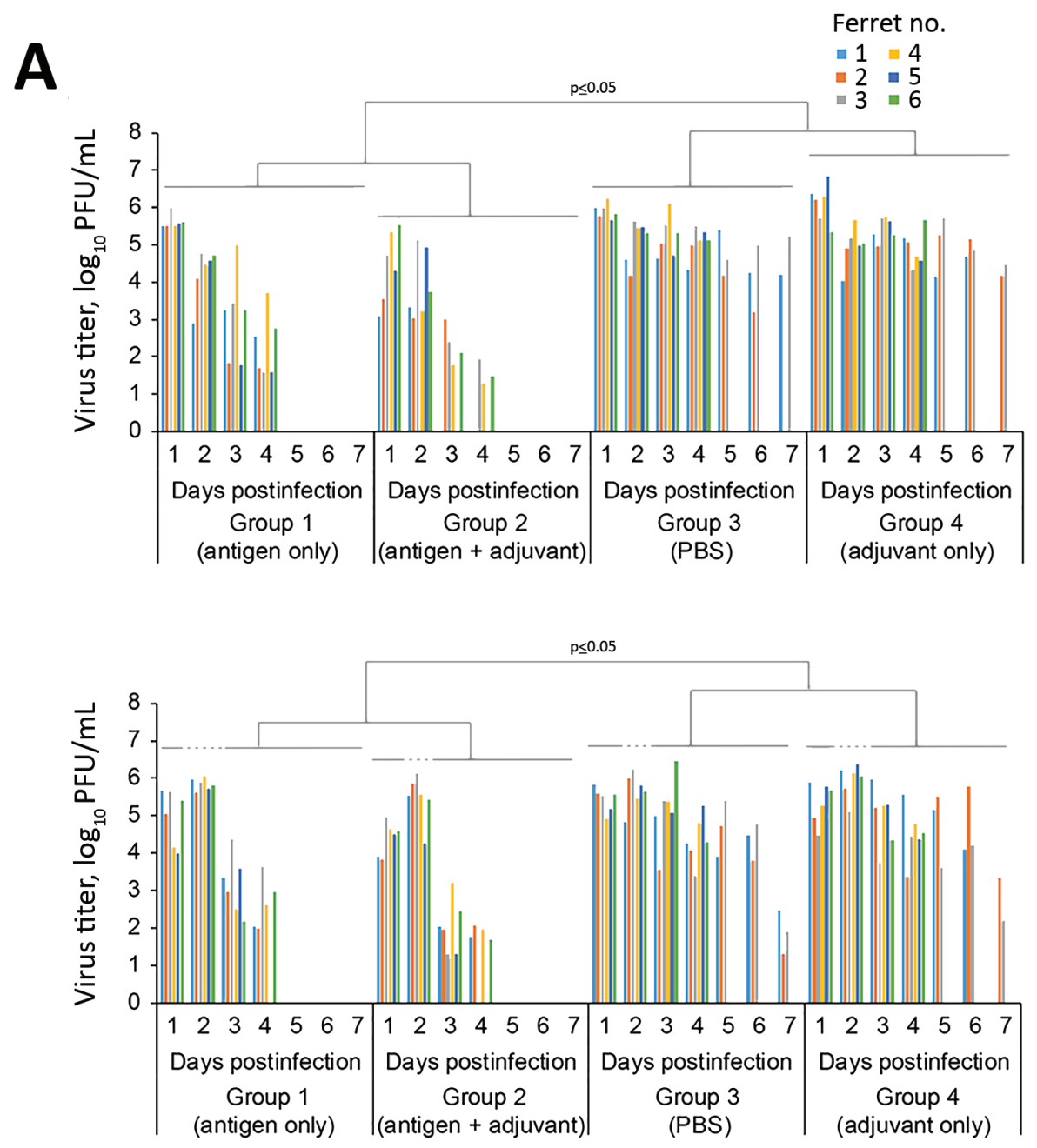

Figure 2. Virus titers in throat and nasal swab specimens and in the organs of vaccinated and nonvaccinated ferrets challenged with highly pathogenic influenza $A(H 7 N 9)$ virus. A) Virus titers in swab samples. Throat and nasal swabs were collected on days 1-7 postchallenge. Virus titers were determined based on plaque assays in MDCK cells. Statistical significance was determined as described in the online Technical Appendix (https://wwwnc.cdc. gov/EID/article/24/10/18-0403Techapp1.pdf). B) Three ferrets from each group were euthanized on day 4 postchallenge for virus titration in the indicated organs. We also assessed virus titers in organs of ferrets that were euthanized because of severe symptoms ( $\left.{ }^{*}\right)$. Virus titers were determined based on plaque assays in MDCK cells. Numbers along baseline indicate animal number. PBS, phosphatebuffered saline.

B

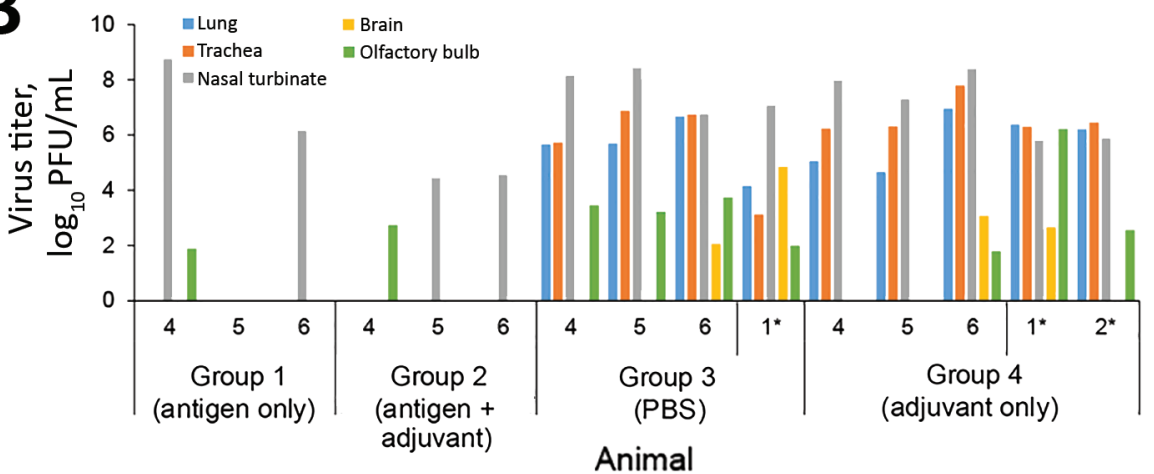


challenge virus were lower than those against the HK125HYPR8 vaccine virus. Statistical analyses demonstrated that HI titers against the HK125-HYPR8 vaccine virus after the first immunization were significantly higher $(\mathrm{p}=$ 0.038 ) in animals immunized with adjuvanted vaccine compared with animals immunized with nonadjuvanted vaccine (Figure 1, panel B; online Technical Appendix Table 2). Bodyweight changes after challenge were significantly milder ( $p=0.0132-0.0489$ on days $4-10,12$, and 13) in ferrets immunized with adjuvanted vaccine than in those vaccinated with nonadjuvanted vaccine. In addition, virus titers in nasal swabs on days 3 and 4 postchallenge ( $p=0.0052$ on day $3 ; p=0.0163$ on day 4 ) and in throat swabs on days 1,3 , and $4(p=0.0047$ on day $1 ; p=0.0003$ on days 3 and 4) in ferrets immunized with nonadjuvanted vaccine were significantly higher than in those ferrets immunized with adjuvanted vaccine (online Technical Appendix Tables 9, 11), suggesting superior efficacy with Addavax.

Previously, WHO selected several low pathogenicity H7N9 candidate vaccine viruses, including A/Hong Kong/125/2017 (7). With the emergence of highly pathogenic H7N9 viruses that are antigenically distinct from previously circulating $\mathrm{H} 7 \mathrm{~N} 9$ viruses, $\mathrm{WHO}$ has updated its recommendations, and a candidate vaccine virus for highly pathogenic H7N9 viruses is now available (12). We tested whether in the event of a large-scale outbreak of highly pathogenic $\mathrm{H} 7 \mathrm{~N} 9$ viruses, candidate vaccine viruses to antigenically distinct $\mathrm{H} 7 \mathrm{~N} 9$ viruses might serve as a first line of defense. Our results in ferrets indicate the potential of a whole, inactivated vaccine based on a low pathogenicity H7N9 virus to prevent severe disease with fatal outcome after infection with an antigenically distinct, highly pathogenic H7N9 virus.

\section{Acknowledgments}

We thank Susan Watson for scientific editing and Alexander Karasin, Kelly E. Moore, Zachary Najacht, and Backiyalakshmi Ammayappan Venkatachalam for technical assistance. We thank personnel from the Research Animal Resources Center and the Charmany Instructional Facility, University of WisconsinMadison, for animal care and technical support.

This work was supported by the National Institute of Allergy and Infectious Diseases-funded Center for Research on Influenza Pathogenesis (grant no. HHSN272201400008C), the Japan Initiative for Global Research Network on Infectious Diseases of the Japan Agency for Medical Research and Development (AMED), AMED's Leading Advanced Projects for Medical Innovation, AMED's Research Program on Emerging and Re-emerging Infectious Diseases, and by Grants-in-Aid for Scientific Research on Innovative Areas from the Ministry of Education, Culture, Science, Sports, and Technology of Japan (grant nos. 16H06429, 16K21723, and 16H06434).

\section{About the Author}

Dr. Hatta is a senior scientist at the Influenza Research Institute at the University of Wisconsin-Madison. His research focuses on identifying the molecular determinants of influenza virus pathogenicity, with particular emphasis on the pathogenicity of highly pathogenic influenza viruses.

\section{References}

1. Centers for Disease Control and Prevention (CDC). Asian lineage avian influenza A (H7N9) virus [cited 2018 Feb 28]. https://www.cdc.gov/flu/avianflu/h7n9-virus.htm

2. Shen $Y, L u H$. Global concern regarding the fifth epidemic of human infection with avian influenza A (H7N9) virus in China. Biosci Trends. 2017;11:120-1. http://dx.doi.org/10.5582/ bst.2017.01040

3 Wang X, Jiang H, Wu P, Uyeki TM, Feng L, Lai S, et al. Epidemiology of avian influenza A H7N9 virus in human beings across five epidemics in mainland China, 2013-17: an epidemiological study of laboratory-confirmed case series. Lancet Infect Dis. 2017;17:822-32. http://dx.doi.org/10.1016/ S1473-3099(17)30323-7

4. Zhou L, Ren R, Yang L, Bao C, Wu J, Wang D, et al. Sudden increase in human infection with avian influenza A(H7N9) virus in China, September-December 2016. Western Pac Surveill Response J. 2017;8:6-14. http://dx.doi.org/10.5365/wpsar.2017.8.1.001

5. Iuliano AD, Jang Y, Jones J, Davis CT, Wentworth DE, Uyeki TM, et al. Increase in human infections with avian influenza A(H7N9) virus during the fifth epidemic - China, October 2016-February 2017. MMWR Morb Mortal Wkly Rep. 2017;66:254-5. http://dx.doi.org/10.15585/mmwr.mm6609e2

6. Centre for Health Protection (Hong Kong). Avian influenza report, volume 13, number 42 [cited 2017 Dec 29]. http://www.chp.gov. hk/files/pdf/2017_avian_influenza_report_vol13_wk42.pdf

7. World Health Organization. Summary of status of development and availability of avian influenza $\mathrm{A}(\mathrm{H} 7 \mathrm{~N} 9)$ candidate vaccine viruses and potency testing reagents [cited 2017 Sep 28]. http://www.who. int/influenza/vaccines/virus/candidates_reagents/summary_a h7n9_cvv_20170928.pdf?ua=1

8. Ping J, Lopes TJ, Nidom CA, Ghedin E, Macken CA, Fitch A, et al. Development of high-yield influenza A virus vaccine viruses. Nat Commun. 2015;6:8148. http://dx.doi.org/10.1038/ncomms9148

9. Ott G, Radhakrishnan R, Fang J-H, Hora M. The adjuvant MF59: a 10-year perspective. In: O’Hagan DT, editor. Vaccine adjuvants: preparation methods and research protocols. Totowa (NJ): Springer New York; 2000. p. 211-28.

10. Imai M, Watanabe T, Kiso M, Nakajima N, Yamayoshi S, Iwatsuki-Horimoto K, et al. A highly pathogenic avian H7N9 influenza virus isolated from a human is lethal in some ferrets infected via respiratory droplets. Cell Host Microbe. 2017;22:615626.e8. http://dx.doi.org/10.1016/j.chom.2017.09.008

11. Zoonotic influenza viruses: antigenic and genetic characteristics and development of candidate vaccine viruses for pandemic preparedness [in French]. Wkly Epidemiol Rec. 2017;92:129-44.

12. World Health Organization. Summary of status of development and availability of avian influenza A(H7N9) candidate vaccine viruses and potency testing reagents [cited 2018 Mar 5]. http://www.who.int/influenza/vaccines/virus/candidates_reagents/ summary_a_h7n9_cvv_20180305.pdf?ua=1

Address for correspondence: Yoshihiro Kawaoka, University of Wisconsin-Madison, Influenza Research Institute, Department of Pathobiological Sciences, School of Veterinary Medicine, 575 Science Dr, Madison, WI 53711, USA; email: yoshihiro.kawaoka@wisc.edu 